\title{
Implication des acides gras trans alimentaires chez la femme enceinte
}

\section{The implications of trans fatty acids for pregnant women}

Oléagineux, Corps Gras, Lipides. Volume 7, Numéro 1, 50-3, Janvier - Février 2000, Dossier : actes des Journées Chevreul "Corps gras, nutrition et santé, questions d'actualité" (Bordeaux, Pessac)

Auteur(s) : Claude BILLEAUD, Unité de néonatalogie et de nutrition pédiatrique, Hôpital Pellegrin, place Emilia-Raba-Léon, 33076 Bordeaux Cedex, France.

Keywords : trans fatty acids, pregnancy, neonate.

\section{ARTICLE}

\section{Matériels et méthodes}

Recueil des paramètres anthropométriques et échantillons de sang et de cordon ombilical

Les 90 parturientes, sans pathologie, ont donné naissance par césarienne programmée à 90 nouveau-nés à terme bien portants. Lors de la césarienne, au niveau du cordon ombilical ont été prélevés du sang veineux ainsi que 4 à $5 \mathrm{~cm}$ de cordon. Divers paramètres ont été relevés chez le bébé (poids, périmètre crânien...).

Analyse du sang et des tissus du cordon

Après prélèvement, les échantillons biologiques ont été conservés à $+4{ }^{\circ} \mathrm{C}$ jusqu'à analyse. La veine et les deux artères du cordon ont été isolées, rincées avec une solution de $\mathrm{NaCl}(0,9 \%)$, puis broyées dans un mélange chloroforme/méthanol $(2 / 1 ; \mathrm{v} / \mathrm{v})$ pour en extraire les lipides. Le plasma et les globules rouges du sang ombilical ont été séparés, les lipides en ont été extraits et analysés selon la méthode précédemment décrite [4].

\section{Analyse statistique}

Les analyses statistiques ont été obtenues par le logiciel SPSS : statistiques descriptives, analyse de la distribution, analyses comparatives et corrélations. Le seuil de significativité retenu était de $p<0,05$.

\section{Résultats et discussion}

Relation, chez la mère, entre consommation et teneur en AGT du plasma

Comme cela a été démontré, la composition du tissu adipeux est un bon reflet de la consommation en AGT. Celle de notre population a été estimée à $3 \mathrm{~g} / \mathrm{j} /$ personne, en moyenne. Durant la grossesse, il y a une augmentation de la lipolyse des triglycérides du tissu adipeux [5] qui contribue à l'élévation du taux de lipides circulants. La figure 1 montre la relation que nous avons mise en évidence entre les taux (en \% des acides gras totaux) d'AGT présents dans le tissu et ceux présents dans le plasma 
maternel. En moyenne, ces taux d'AGT sont respectivement égaux à 2,3 et $0,7 \%$. Plus il y a d'AGT (ou de 18:1 trans : isomère majoritaire) incorporés dans le tissu adipeux, plus il y en a dans le sang ( $\mathrm{p}<$ $0,001)$.

L'alimentation apporte principalement l'isomère 18:1 trans [6]. C'est en effet ce que l'on constate au travers de la composition du tissu adipeux, où $87 \%$ des AGT sont représentés par le 18:1t, $10 \%$ par les isomères trans de l'acide linoléique et $3 \%$ par le 16:1t. II est important de remarquer que ces proportions diffèrent dans le plasma : les isomères trans de l'acide linoléique, susceptibles d'interférer dans le métabolisme des AGE [7, 8], y représentent $24 \%$ des AGT totaux contre $10 \%$ dans le tissu adipeux. C'est donc dans ces proportions que les AGT parviennent au placenta.

Passage des isomères trans de la mère au fotus

Le pourcentage d'AGT totaux (figure 2) dans le sang du cordon $(0,60 \% \pm 0,18)$ n'est pas significativement différent de celui du sang maternel $(0,65 \% \pm 0,17)$, chez 31 couples mère/enfant. Ces valeurs sont 3 fois plus faibles que celles rapportées par Koletzko [9] pour la population allemande. On observe chez le fœtus les mêmes isomères que chez la mère, mais leurs proportions respectives sont différentes. Ainsi, le sang du cordon contient moins de monoènes mais plus de diènes trans, isomère de l'acide linoléique (18:2 9c, $12 \mathrm{c}$ ) essentiel, en particulier trois fois plus ( $p<$ $0,001) d$ disomère 18:2 9t, 12c. On observe également une discrimination entre les isomères 18:2 9t, $12 c$ et 18:2 9c, 12t. Le rapport 18:2 9t, 12c/18:2 9c, 12t est de 0,8 dans le plasma maternel contre 2,3 dans le plasma du cordon. II existe donc un passage sélectif transplacentaire des AGT. Le taux plus élevé de 18:2 tc dans le sang du cordon pourrait être lié à un passage transplacentaire sélectif ou bien à un métabolisme plus lent de cet isomère chez le fœtus.

L'indice d'échange placentaire (EI) de chaque isomère trans par rapport à l'acide linoléique ( $\mathrm{AL}$ ) a été calculé à partir de leurs teneurs respectives dans le plasma du cordon, d'une part, et dans le plasma maternel, d'autre part :

$\mathrm{EI}=(\% \mathrm{AGT}$ cordon $/ \% \mathrm{AL}$ cordon $) /(\% \mathrm{AGT}$ mère $/ \% \mathrm{AL}$ mère $)$ - 1.

Si $E I=0$, il n'y a pas de différence d'incorporation dans le cordon entre l'isomère trans et l'acide linoléique ; si $\mathrm{El}<0$, l'acide linoléique est mieux incorporé dans le cordon que l'isomère trans ; si El > 0 , l'isomère trans est mieux incorporé dans le cordon que l'acide linoléique.

On constate que l'isomère 18:2 9t, 12c s'incorpore dans le cordon préférentiellement à son isomère naturel cis, l'acide linoléique essentiel (IE =6). Ce comportement n'est pas observé avec les autres isomères. Ce qui pourrait s'expliquer par des différences dans l'utilisation métabolique. II est bien connu que l'isomère $9 t, 12 c$ est métabolisé plus lentement que l'acide linoléique $(9 c, 12 c)$ et que l'isomère $9 c, 12 t$, dans la synthèse des acides gras polyinsaturés à longue chaîne [10].

Incorporation des isomères trans dans le plasma du cordon

Les AGT ont été retrouvés dans toutes les classes de lipides plasmatiques du cordon (acides gras libres, triglycérides, esters de cholestérol, phospholipides), mais en proportions variables. En particulier, l'acide 18:2 9t, 12c est l'isomère majoritaire dans les esters de cholestérol (EC) qui représentent une fraction lipidique d'intérêt pour leur rôle dans le transport des AGE vers le fœtus. Question cruciale : est-ce que les isomères trans interfèrent avec le métabolisme des AGE ? Koletzko 
[2] a rapporté que, dans les EC du plasma du cordon, le taux de 18:1 trans était corrélé négativement à celui d'acide arachidonique. Decsi [11] a montré que, chez l'enfant plus grand, le contenu en AGT du plasma était inversement corrélé aux acides gras à longues chaînes. La figure 3 illustre les différences que nous avons observées entre les teneurs en AGE et AGT des EC du plasma maternel et celles du plasma ombilical. Comme attendu, le pourcentage d'acide linoléique est plus faible dans le cordon ; c'est l'inverse pour les longues chaînes : 20:4 n-6 et 22:6n-3. Le taux d'AGT y est trois fois plus élevé que chez la mère, ce qui est dû principalement à l'incorporation de l'isomère $9 t, 12 c$. II existe une corrélation négative entre le taux de 18:2 9t12c et l'acide linoléique dans les EC du plasma ombilical $(r=0,696 ; p<0,001 ; n=29)$. En revanche, aucune relation n'est apparue avec le 18:1t, comme dans l'étude de Koletzko [2].

Incorporation des isomères trans dans les tissus du cordon

La figure 4 représente les divers isomères trans trouvés dans les globules rouges, la veine et les artères du cordon $(n=10)$. Le taux d'AGT y est respectivement de $0,25,0,34$ et $0,40 \%$ par rapport aux acides gras totaux. Les proportions des divers isomères trans y sont identiques, à l'exception de I'isomère 18:2 9t, 12t qui prédomine dans les artères. Cet isomère pourrait être accompagné d'un autre isomère, non séparé du $9 \mathrm{t}, 12 \mathrm{t}$ et qui serait le $9 \mathrm{c}, 13 \mathrm{t}$. Ces résultats appellent les remarques suivantes :

- l'isomère 18:2, majeur dans les lipides circulants, ne se concentre pas dans les tissus analysés ;

- les vaisseaux et plus précisément les artères privilégient l'isomère 9t, 12t. Ceci pourrait être un effet du métabolisme fœtal vis-à-vis de cet isomère, dans la mesure où les artères drainent le sang efférent au fœtus. Compte tenu de la faible taille $(n=10)$ de l'échantillon et de l'effet potentiel négatif de cet isomère sur le métabolisme de l'acide linoléique, ces résultats devront être confirmés.

Par ailleurs, une corrélation positive a été mise en évidence entre le taux de 18:2 trans et celui de C20:3n-9 (marqueur classique de la déficience en AGE) dans les phospholipides des artères du cordon ( $r=0,824 ; p<0,005 ; n=10)$, comme si les AGT altéraient le métabolisme de l'acide linoléique.

\section{Consommation en AGT de la mère et paramètres anthropométriques du nouveau-né}

L'incidence de la consommation en AGT de la mère sur le développement du fœetus a été étudiée au travers d'analyses de corrélations entre, d'une part, les teneurs en AGT du tissu adipeux et du plasma de la mère et, d'autre part, le poids et le périmètre crânien du nouveau-né.

Aucune relation n'a été observée comme en témoignent les figures 5 et 6 , contrairement à ce qui fut rapporté pour l'Allemagne [2] et la Pologne [3].

\section{CONCLUSION}

Cette étude a confirmé le passage des AGT de la mère au fœtus. En outre, elle a mis en évidence l'incorporation des divers isomères trans dans les tissus du cordon ombilical. L'analyse statistique des données montre que la consommation en AGT des femmes parturientes étudiées en Aquitaine est sans incidence sur le poids et le périmètre cranien du nouveau-né. 


\section{REFERENCES}

1. KUHN DC, CRAWFORD M (1986). Placental essential fatty acid transport and prostaglandin synthesis. Prog Lipid Res, 25 : 345-53.

2. KOLETZKO B (1992). Trans fatty acids may impair biosynthesis of long-chain polyunsaturated and growth in man. Acta Paediatr, 81 : 302-6.

3. JENDRYCZKO A, GRUSZCZYNSKI J, TOMALA T, et al. (1993). Unsaturated fatty acids of trans isomers in plasma of pregnant women and newborn birth weight. Ginekol Pol, 64 : 113-6.

4. COMBE N, JUDDE A, BILLEAUD C, BOUE C, TURON F, ENTRESSANGLES B, DALLAY D, LENG JJ (1998). Distribution of dietary trans isomers of essentiel fatty acids in blood lipid classes. In : RIEMERSMA RA, AMSTRONG RA, KELLY RW, WILSON R, eds. Proceedings of the Fourth International Congress on Essential Fatty Acids and Eicosanoids. Ville : AOCS Press : 239-42.

5. HERRERA E, GOMEZ-CORONADO D, LASUNCION MA (1987). Lipid metabolism in pregnancy. Biol Neonate, $51: 70-7$.

6. COMBE N (1996). Oils \& Fats. Paris : Lavoisier, 652-62.

7. ENTRESSANGLES B (1995). Isomères trans d'acides gras insaturés : aspects métaboliques et nutritionnels. OCL, $2: 162-9$.

8. CARLSON SE, CLANDININ MT, COOK HW, EMKEN EA, FILER L (1997). Trans fatty acids : infant and fetal development. Am J Clin Nutr, 66 : S717-36.

9. KOLETZKO B, MÜLLER J (1990). Cis- and Trans-isomeric fatty acids in plasma lipids of newborn infants and their mothers. Biol Neonate, $57: 172-8$.

10. BERDEAUX O, BLOND JP, BRÉTILLON L, et al. (1998). In vitro desaturation or elongation of monotrans isomers of linoleic acid by rat liver microsomes. Mol Cell Biochem, 185 : 17-25.

11. DECSI T, KOLETZKO B (1995). Do trans fatty acids impair linoleic acid metabolism in children ? Ann Nutr Metab, 39 : 36-41. 
Illustrations

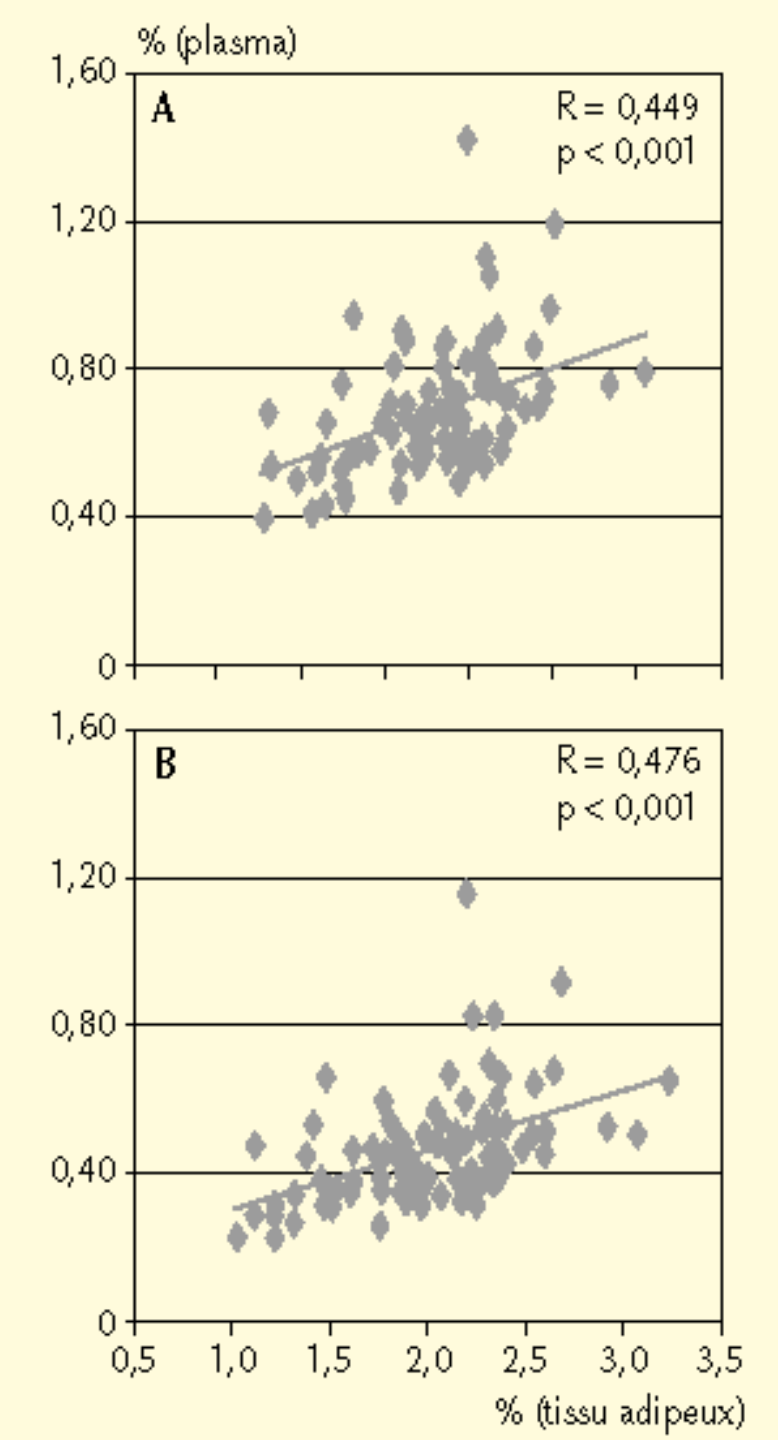

Figure 1. Relations entre les taux d'acides gras trans présents dans le plasma et dans le tissu adipeux de femmes parturientes $(n=90)$. A : acides gras trans totaux. $B$ : isomères 18:1 trans. 


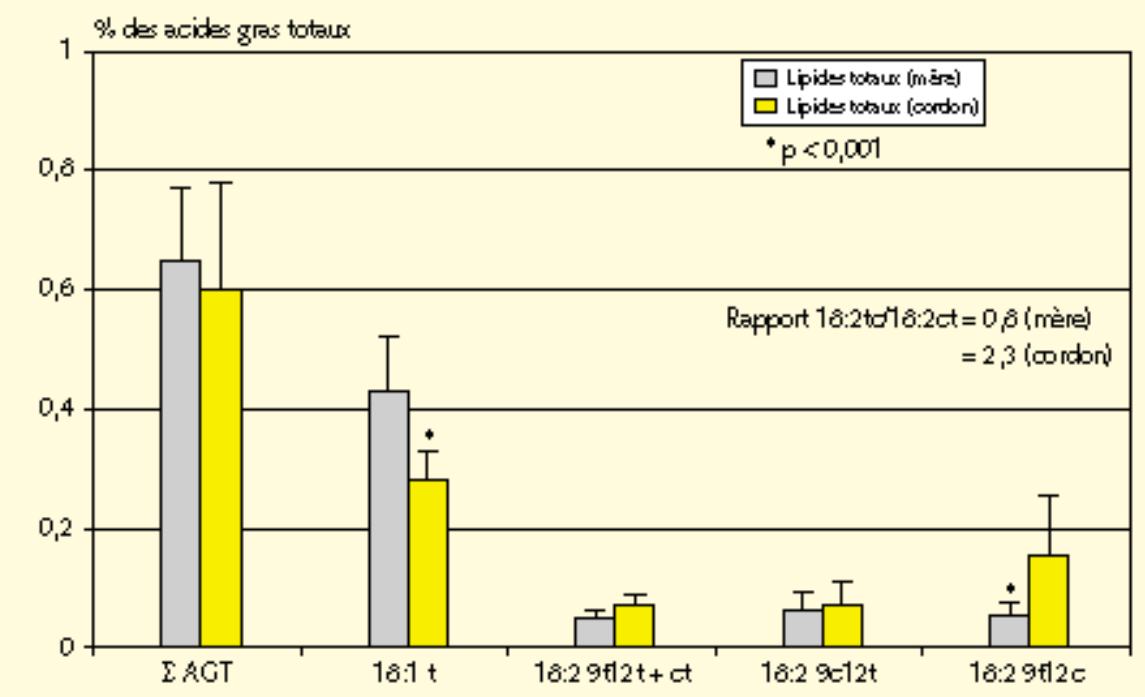

Figure 2. Composition (\%) en acides gras trans du plasma maternel et du plasma du cordon ombilical ( $n=31$ couples). 

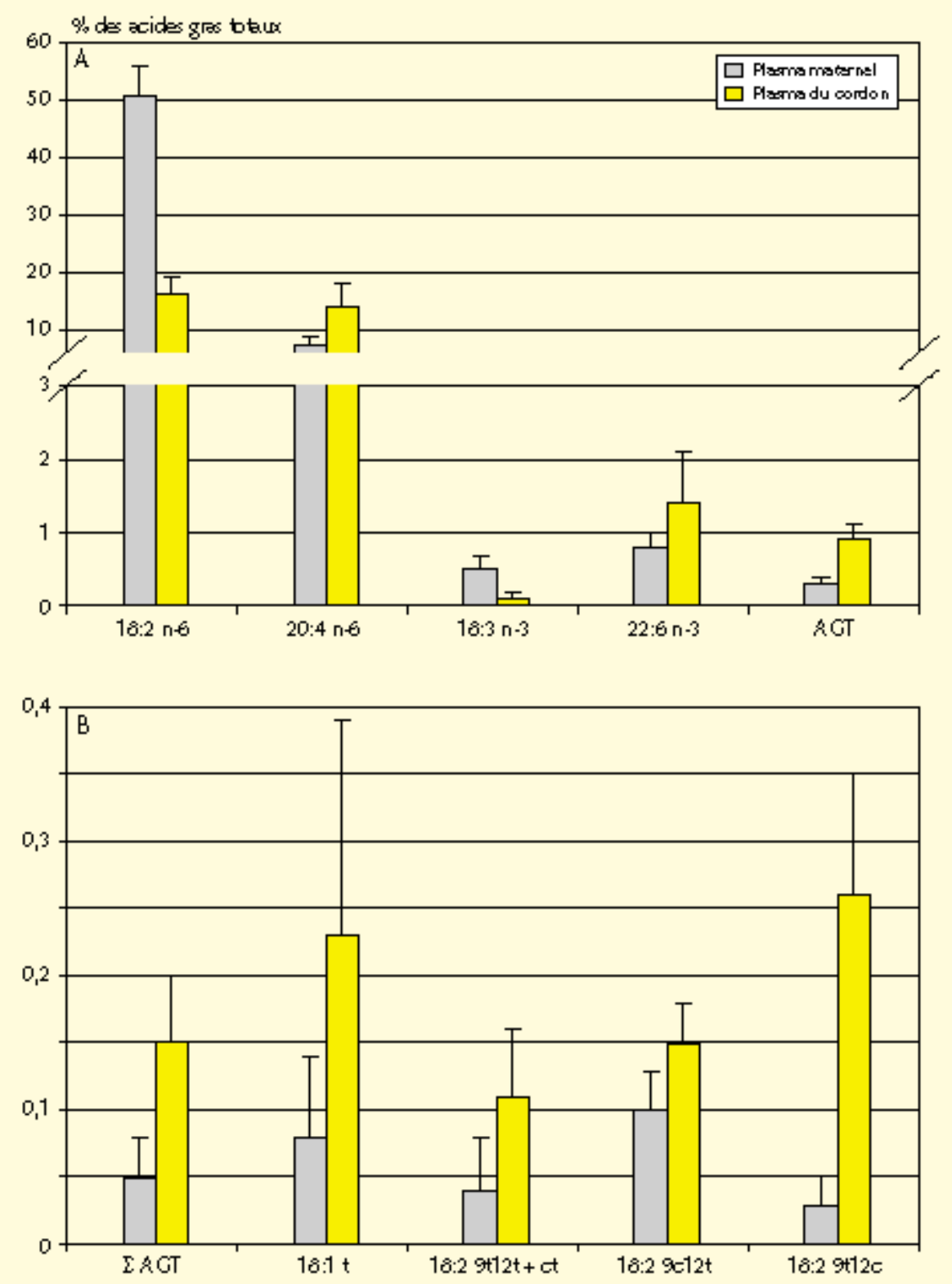

Figure 3. Composition (\%) en acides gras essentiels (AGE) et acides gras trans (AGT) des esters de cholestérol (mère versus cordon) ( $n=29$ couples). $A$ : AGE et AGT. B : isomères trans. 


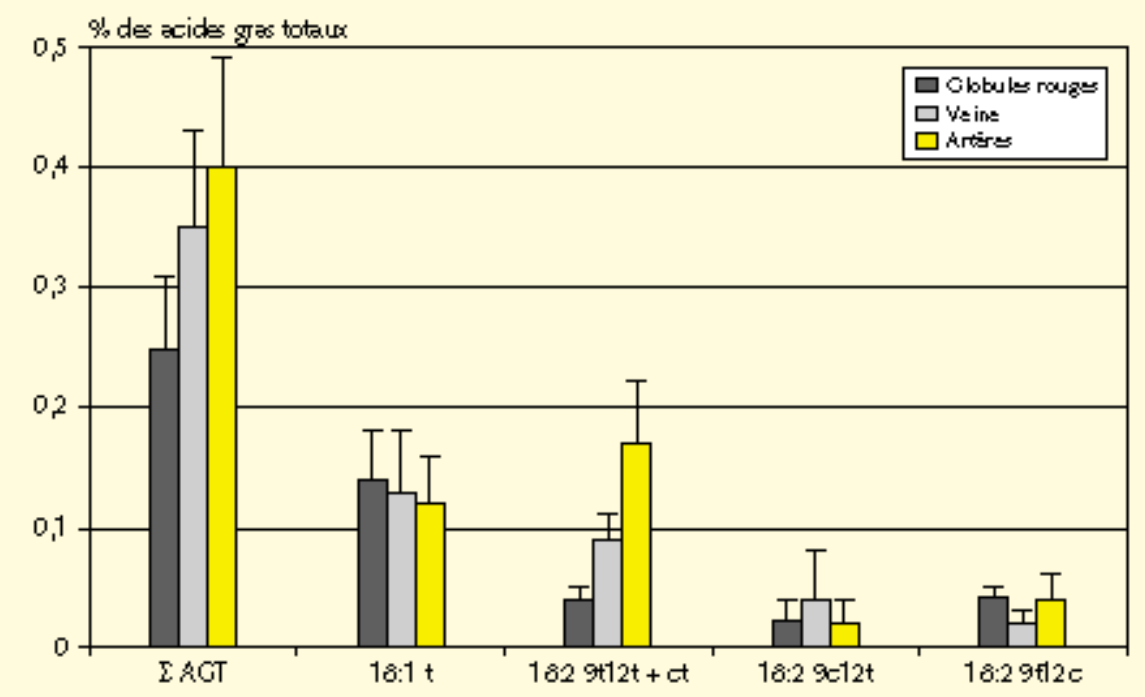

Figure 4. Composition (\%) en acides gras trans des globules rouges, veine et artères $d u$ cordon ombilical $(n=10)$.
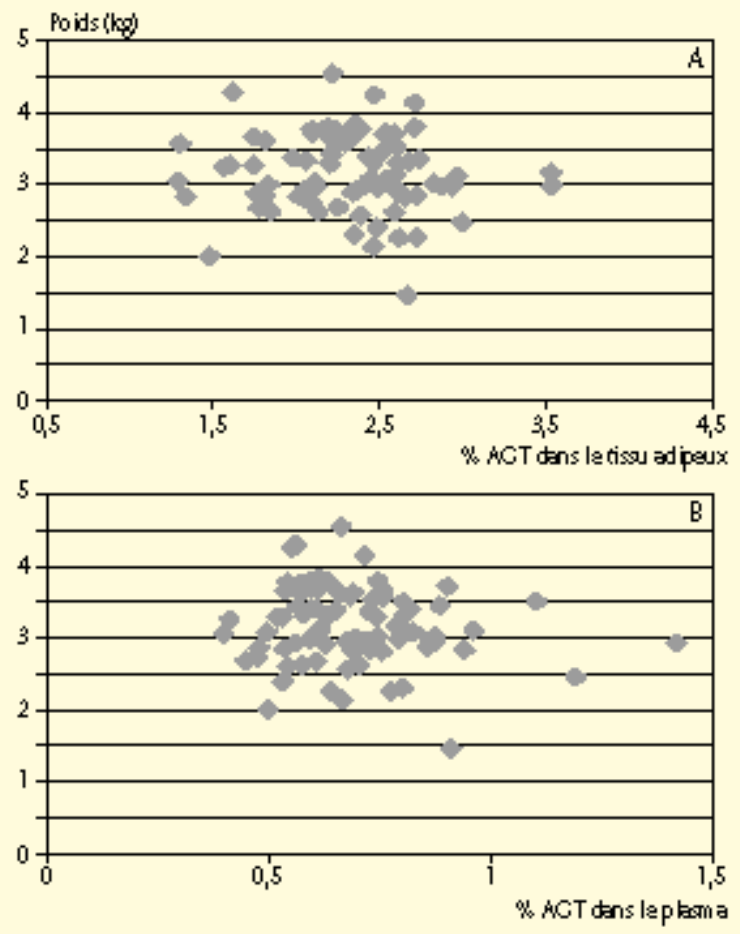

Figure 5. Poids du nouveau-né et taux d'acides gras trans dans le tissu adipeux (A) et le plasma $(B)$ de la mère ( $n=81$ couples). 

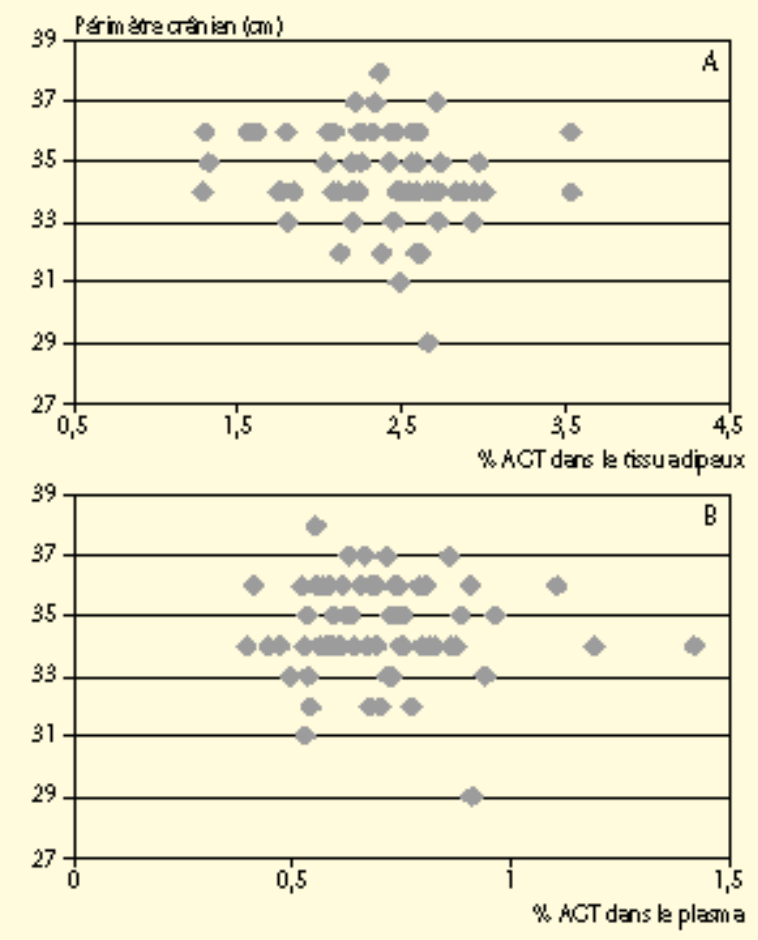

Figure 6. Périmètre crânien $P C$ du nouveau-né et taux d'acides gras trans dans le tissu adipeux $(A)$ et le plasma $(B)$ de la mère ( $n=68$ couples). 